\section{Exposing children to secondhand smoke}

In the October issue of Thorax, Leonardi-Bee et al reviewed the effects of parental smoking on the uptake of smoking by children. ${ }^{1}$ They conclude that exposure to smoking within the family is a significant determinant of subsequent smoking.

One area that this study did not address was the effect of the site of the smoking on exposure.

We have studied urinary cotinine levels in children attending an asthma clinic and correlated them with the self-reported smoking habits of their carers. Thirty-six girls and 64 boys aged between 30 and 164 months attending our asthma clinic were recruited if at least one carer smoked. The carers completed a questionnaire about their smoking habits and the child's urine was tested for cotinine using NicAlert, a commercially available reagent strip. Levels of $0-10 \mathrm{ng} / \mathrm{ml}$ correspond to minimal or no nicotine exposure, $10-100 \mathrm{ng} / \mathrm{ml}$ to moderate to high SHS exposure and $>100 \mathrm{ng} / \mathrm{ml}$ to active smoking. Ten further children were recruited from the same clinic from nonsmoking families and acted as controls.

Seventy-two 'smoking' families (72\%) reported only smoking outside the house ('outside' smokers) while 28 parents (28\%) did not take any harm reduction strategies ('everywhere' smokers). Three (4\%) of the children in outside smoking carers' group had levels indicative of active smoking and were excluded from analysis. Urine testing showed measureable cotinine $(10-100 \mathrm{ng} / \mathrm{ml})$ in 27 of $28(96 \%)$ and 61 of 69 (88\%) everywhere and outside smokers' children, respectively $(p=0.20)$. Only one child in the control group $(10 \%)$ had detectable cotinine $(p<0.0001$, compared with both everywhere and outside smokers' children) and it transpired that she was exposed to SHS at school.

Our findings suggest that there is little or no difference between nicotine exposure in those children of outside smokers and those in direct contact with smokers (everywhere smokers). Even if a proportion of our outside smokers were in fact smoking indoors, the very high percentage of this group that had raised cotinine levels indicates that most of the 'outdoor' smokers are indeed exposing their children to SHS. This is consistent with reports previously published, showing that nicotine can be transmitted through a number of third hand routes. ${ }^{2}$

Harm reduction strategies (effectively limiting the exposure) are reported to have some benefit in reducing nicotine exposure although our data suggest that reduction may have been overestimated in the past. ${ }^{3}$ Using urinary cotinine levels may also be useful in helping advice on smoking cessation. ${ }^{4}$ In our study we were able to use the results which were shown to parents at the time of the clinic, and they did seem to focus parental attention on the problem. We found that a number of parents reported cessation of smoking following the test and that their children were free of urinary cotinine when tested.

We suggest that measurement of urinary cotinine can be a useful adjunct when discussing parental smoking and that outside smoking may not protect children from SHS exposure as much as previously thought.

\section{Jenny Pool, ${ }^{1}$ Natalya Petrova, ${ }^{2}$ Robert Ross Russell}

${ }^{1}$ Department of Paediatrics, Cambridge University Hospitals NHS Foundation Trust, Cambridge, UK: ${ }^{2}$ Department of Neonatal, Almazov Federal Heart, Blood and Endocrinology Centre, St Petersburg, Russian Federation

Correspondence to Jenny Pool, Department of Paediatrics, Cambridge University Hospitals NHS Foundation Trust, Cambridge, UK; jenny.pool@ addenbrookes.nhs.uk

Contributors JP and RIRR developed the concept for the study, recruitment and data collection. The letter was composed by all three authors.

Competing interests None.

Ethics approval Ethics approval was provided by Cambridge Local Research Ethics Committee.

Provenance and peer review Not commissioned; internally peer reviewed.

Data sharing statement All data from this study are available to anyone interested. Please contact the authors for further details.

Accepted 12 January 2012

Published Online First 10 February 2012

Thorax 2012;67:926.

doi:10.1136/thoraxjnl-2012-201607

\section{REFERENCES}

1. Leonardi-Bee J, Jere ML, Britton J. Exposure to parental and sibling smoking and the risk of smoking uptake in childhood and adolescence: a systematic review and meta-analysis. Thorax 2011;66:847-55.

2. Matt GE, Quintana PJ, Hovell MF, et al. Households contaminated by environmental tobacco smoke: sources of infant exposures. Tob Control 2004;13:29-37.
3. Spencer N, Blackburn C, Bonas S, et al. Parent reported home smoking bans and toddler (18-30 month) smoke exposure: a cross-sectional survey. Arch Dis Child 2005;90:670-4.

4. Irvine L, Crombie IK, Clark RA, et al. What determines levels of passive smoking in children with asthma? Thorax 1997;52:766-9. 